\title{
miRNA-16-5p inhibits the apoptosis of high glucose-induced pancreatic $\beta$ cells via targeting of CXCL10: potential biomarkers in type 1 diabetes mellitus
}

\author{
Xiaoyan Gao', Shumiao Zhao ${ }^{2}$ \\ ${ }^{1}$ Department of Internal Medicine, Tianqiao District People's Hospital of Jinan City, Jin'an City, Shandong Province, China \\ ${ }^{2}$ Department of Endocrinology, The Fifth's People's Hospital of Ji'nan City, Ji'nan City, Shandong Province, China
}

\begin{abstract}
Introduction: We aimed to elucidate the relationship between CXC chemokine ligand 10 (CXCL10) and miR-16-5p, and their functions on the biological behaviour of type 1 diabetes mellitus (T1DM).

Material and methods: The GSE72492 dataset from the GEO database was used to analyse gene expression. We discovered that CXCL10 was highly expressed in T1DM patients. The up-stream miRNA was predicted by Targetscan website. Low glucose $(2.8 \mathrm{mmol} / \mathrm{L})$ and high glucose (HG, $16.7 \mathrm{mmol} / \mathrm{L}$ ) were utilised to treat $\beta$-TC-tet (pancreatic $\beta$ cell) cells to form the model. The direct interaction between miR16-5p and CXCL10 was verified by a dual-luciferase reporter assay. Real-time quantitative PCR (qRT-PCR) and western blotting analyses were used to detect RNA and protein expression. CCK8 and flow cytometry were used to detect cell proliferation and apoptosis.

Results: We discovered that CXCL10 was highly expressed in T1DM patients. MiR-16-5p, which was lowly expressed in T1DM patients, was verified the upstream regulatory miRNA of CXCL10. The facilitating influence of miR-16-5p up-regulation on the proliferation of HG-induced $\beta$-TC-tet cells was reversed by CXCL10 over-expression, while the knockdown results were opposite. More importantly, the restraining impact of miR-16-5p high expression on the apoptosis of HG-induced $\beta$-TC-tet cells was accelerated by CXCL10 over-expression. Correspondingly, the level of Bcl-2 was enhanced while the levels of Bax and Cleaved Caspase- 3 were lowered by miR-16-5p mimic, which were reversed by CXCL10 over-expression in HG-treated $\beta$-TC-tet cells.

Conclusions: Our data offered evidence that miR-16-5p implicated in T1DM cell proliferation and apoptosis through targeting CXCL10, which might provide novel therapeutic information for T1DM. (Endokrynol Pol 2020; 71 (5): 404-410)
\end{abstract}

Key words: type 1 diabetes mellitus; CXC chemokine ligand 10; miRNA-16-5p; target; relationship

\section{Introduction}

Diabetes, with the basic characteristic of hyperglycaemia, is a chronic metabolic disease and one of the most challenging heterogeneous diseases, which can be briefly divided into type 1 diabetes mellitus (T1DM) and T2DM [1,2]. Type 1 diabetes mellitus is a chronic autoimmune disease that is featured by the destruction of islet $\beta$-cells, ultimately leading to insulin deficiency [3, 4]. The pathogenesis of T1DM is complex and may be caused by complex interactions between environmental and genetic factors, just like other autoimmune diseases [5]. Insulin treatment is not enough to improve the prognosis of patients with T1DM [6]. There are many complications of the disease, such as stroke, blindness, some vascular problems, heart disease, and kidney diseases [7]. Precise supervising of the advance of these complications and early responses to treatment will help to improve prevention and treatment strategies for patients with diabetes [8]. However, the pathological mechanism of T1DM is not clear and requires further study. Moreover, finding better markers and therapeutic targets will help predict potential complications and improve our ability to prevent and treat T1DM.

Due to the fact that CXC chemokine ligand 10 (CXCL10) expression is related to the incidence of T1DM, it has drawn lots of research interest regarding the impact of CXCL10 on T1DM [9]. CXCL10, as a pro-inflammatory cytokine, is concerned with various biological processes, including modulation of cell growth, apoptosis, activation and chemotaxis of peripheral immune cells, and regulation of angiostatic effects, which are consistent with the known effects of CXC cytokines [10,11]. CXC chemokine ligand 10 is indicated to modulate immune responses by recruiting leukocytes, containing monocytes, $\mathrm{T}$ cells, and macrophages [12]. CXC chemokine ligand 10 belongs to a large $\mathrm{CXC}$ subfamily and is a diminutive secretory immuno-modulator [13]. A previous study revealed that CXCL10 appears to be the main chemokine ex- 
pressed by the pancreas in early human T1DM, as well as in a mouse model [14]. In recent years, growing evidence has demonstrated that a highly expression of CXCL10 was discovered in the tissues and/or the serum of multifarious auto-immune diseases such as T1DM [15]. Therefore, CXCL10 is closely associated with the development of T1DM, stimulating our interest to study whether the upstream regulatory factors of this gene also have an influence on T1DM, to further understand the pathological mechanism of this disease.

MicroRNAs (miRNAs) are a series of single-stranded, non-coding small RNAs of 18-25 nt, which modulate the levels of target genes via binding to their 3'UTR [16]. Our paper revealed that miR-16-5p was predicted as an upstream miRNA that regulates CXCL10, by using the Targetscan website. Furthermore, growing studies affirmed that some miRNAs are associated with various biological processes in T1DM [17, 18]. Most importantly, as reported by Assmann et al. [19], miR-16-5p was dysregulated in T1DM patients in diabetic kidney disease of different stages through silico analyses. Nevertheless, the potential of miR-16-5p as a regulator for CXCL10 and as a biomarker for the development of T1DM has not been sufficiently exploited.

The present study showed that CXCL10 was highly expressed and miR-16-5p was lowly expressed in T1DM patients. Moreover, miR-16-5p was predicted and verified as an upstream miRNA that regulates CXCL10, for the first time. In addition, miR-16-5p, which might target CXCL10, facilitated the proliferation and suppressed the apoptosis of high glucose-treated $\beta$-TC-tet cells. Above all, our study showed the targeting relationship between CXCL10 and miR-16-5p for the first time, which might offer a theoretical basis for the diagnosis and treatment of T1DM.

\section{Material and methods}

\section{Data analysis of GEO database}

Tissues of T1DM patients $(n=10)$ and control specimens $(n=7)$ were utilised to analyse the expression of CXCL10 in TIDM, which were obtained from the GEO database (no.GSE72492; http://www. ncbi.nlm.nih.gov/geo). Then we predicted the upstream regulatory miRNA of CXCL10 by employing the miRNA target gene prediction website Targetscan (http://www.targetscan.org). The common miRNAs were obtained from the intersection between miRNAs predicted by Targetscan website and the down-regulated miRNAs in T1DM that were acquired on the basis of the GEO database (no. GSE97123). The miR-16-5p expression data (including 12 T1DM tissues and 12 control samples) were acquired from the GEO database, with access number GSE97123.

\section{Cell cultivation}

$\beta$-TC-tet (mouse pancreatic $\beta$ cell) cells were obtained from the American Type Culture Collection (ATCC, USA). The cells were cultivated in DMEM medium containing 10\% FBS and were divided into three groups: low glucose $(2.8 \mathrm{mM})$, high glucose $(16.7 \mathrm{mM})$, and control (without adding glucose). When the cells achieved $80-90 \%$ confluence, the medium was discarded. Then $0.25 \%$ trypsin was added to the cells to digest for about $30 \mathrm{~s}$ at $37^{\circ} \mathrm{C}$, and $10 \%$ FBS medium was added to terminate the digestion. A single-cell suspension was formed by pipetting the cells gently. Then the cell suspension was centrifuged at $1000 \mathrm{rpm}$ for five minutes and discarded the supernatant. Subsequently, the cells were re-suspended and cultured in a 3:1 ratio, and the culture medium was replaced every two days.

\section{Cell transfection}

Synthetic miR-16-5p mimic/inhibitor and their corresponding negative control (NC), pcDNA3.1-CXCL10, pcDNA3.1-, si-CXCL10 (5'-AAGACAATGTACTGTATTGAAAG- $\left.3^{\prime}\right)$, and si-con (5'-TGAATCATGTTTCAATGTTCATC-3') were acquired from Dharmacon (Boulder, CO). Then they were transfected into $\beta$-TC-tet cells utilising Lipofectamine 3000 reagent (Invitrogen). After 24 hours, the transfected efficiency was determined by qRT-PCR.

\section{qRT-PCR}

Total RNA was extracted using TRIzol (Invitrogen). By employing the PrimeScript RT Reagent Kit or the Mir- ${ }^{\mathrm{TM}}$ miRNA First Strand Synthesis Kit, reverse transcription was conducted. SYBR Premix Ex Taq II and SYBR PrimeScriptTM miRNA RT-PCT Kit were applied to detect CXCL10 and miR-16-5p expression in an AB7300 real-time PCR machine. GAPDH was utilised as an internal reference for CXCL10 detection. U6 served as a reference for miR-16-5p detection. $2^{-\Delta \Lambda C t}$ was employed to analyse the levels of CXCL10 and miR-16-5p. Table 1 displayed the primers used for qPCR.

\section{Western blotting}

Cells were lysed utilising RIPA buffer (including a protease inhibitor cocktail), and the concentration was measured by BCA assay. Protein sample was isolated by SDS-PAGE, shifted onto a PVDF membrane, and then blocked in 5\% skimmed milk for one hour. Subsequently, the membrane was incubated with primary antibodies at $4^{\circ} \mathrm{C}$ overnight. After washing with PBS three times, the membrane was probed with a second HRP antibody at room temperature for two hours. Ultimately, the proteins were tested by ECL (Millipore, USA).

\section{Analysis of cell proliferation}

Cell proliferation was tested utilising a Cell Counting Kit-8 (CCK8) (Beyotime, China) following the manufacturer's instructions. Briefly, $\beta$-TC-tet cells were seeded in a 96-well plate at about 1000 cells/well and cultivated with complete DMEM medium. At 0, 24, 48 , and $72 \mathrm{~h}$ time points, $10 \mu \mathrm{L}$ of CCK8 reagent was put into each well and incubated for $1.5 \mathrm{~h}$ at $37^{\circ} \mathrm{C}$. Finally, the $\mathrm{OD}_{450}$ was detected using a plate reader.

Table 1. The primers used in qRT-PCR

\begin{tabular}{|c|c|}
\hline Name & Sequences \\
\hline miR-16-5p forward & 5'-TAGCAGCACGTAAATATTGG-3' \\
\hline miR-16-5p reverse & 5'-GAACATGTCTGCGTATCTC-3' \\
\hline U6 forward & 5'-CGTATGAGGCGAAGGAGGAC-3' \\
\hline U6 reverse & 5'-GTCCTCCTTCGCCTCATACG-3' \\
\hline CXCL10 forward & 5'-ATCATCCCTGCGAGCCTATCCT-3' \\
\hline CXCL10 reverse & 5'-GACCTTTTTTGGCTAAACGCTTTC-3' \\
\hline GAPDH forward & 5'-САTСАСТGCСАСССАGAAGACTG-3' \\
\hline GAPDH reverse & 5'-ATGCCAGTGAGCTTCCCGTTCAG-3' \\
\hline
\end{tabular}


A

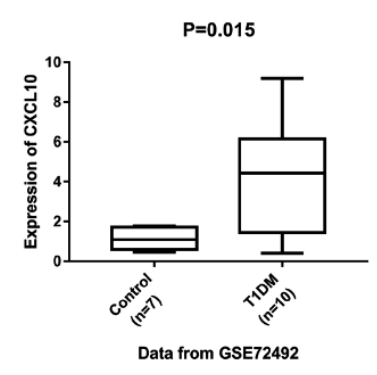

\section{B}

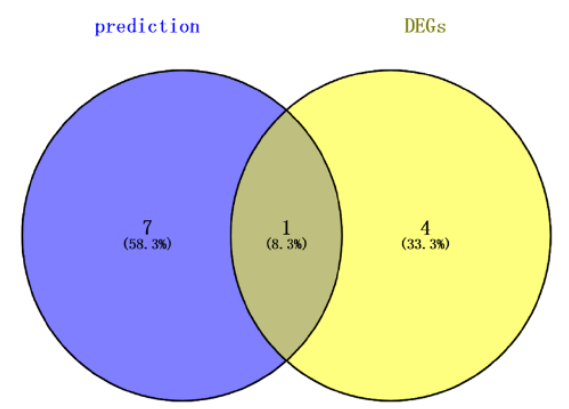

C

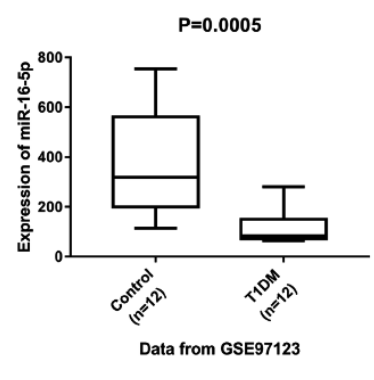

Figure 1. Highly expressed CXCL10 and lowly expressed miR-16-5p in the tissues of T1DM patients. A. The expression level of CXCL10 in T1DM patients was tested in GEO database. B. There was one common miRNA. The blue part was the predicted miRNA. The yellow part was the lowly expressed miRNAs in the GEO database with the number GSE97123. C. According to the data gained from the GEO database, miR-16-5p expression level in T1DM patients and control samples was analysed. T1DM - type 1 diabetes mellitus

\section{Flow cytometry}

The apoptosis level was conducted utilising an Annexin V-FITC/PI Detection Kit and detected by flow cytometry (BD Biosciences) following the manufacturer's directions. Lastly, the results were analysed by FlowJo software (V.10, Treestar, USA).

\section{Dual-luciferase reporter assay}

To construct pGL3-CXCL10-3'UTR-wild type (WT) or pGL3CXCL10-3'UTR-mutant (MUT), a fragment of CXCL10 3'UTR-WT or CXCL10 3'UTR-MUT was amplified and inserted into the pGL3 luciferase reporter vectors (Promega, USA). $\beta$-TC-tet cells were cotransfected with these vectors and miR-16-5p mimic, miR-16-5p inhibitor, or control utilising Lipofectamine 3000 reagent. After 24-h transfection, the Dual-Luciferase Reporter Assay System (Promega, USA) was used to detect luciferase activity following the directions.

\section{Statistics}

Appropriate statistical testing was executed with GraphPad Prism 7.0 and SPSS 22.0 software. Comparisons were carried out utilising Student's t-test (for single comparisons) or ANOVA test with Dunnett post hot (for multiple comparisons). Error bars indicate $\mathrm{SD}$ in the figure legends. Two-sided $\mathrm{p}<0.01$ was regarded as statistically significant.

\section{Results}

High expression of CXCL10 and low expression of miR-16-5p in the tissues of T1DM patients

Herein, to verify the effect of CXCL10 in T1DM, we first analysed the expression of CXCL10 in the public dataset GEO with access number GSE72492. As displayed in Figure 1A, CXCL10 was highly expressed in T1DM tissues $(n=10)$ relative to control specimens $(n=7)$, which was consistent with previous reports. Next, we used Targetscan to predict the upstream miRNAs that regulate CXCL10, from which eight potential up-stream miRNAs were identified (the blue part in Fig. 1B). In addition, five miRNAs were downregulated in T1DM tissues by analysing the GEO dataset (no. GSE97123) (the yellow part in Fig. 1B). The miRNAs that met two requirements: the upstream miRNAs that regulate CXCL10 and were downregulated in T1DM tissues, were selected for further exploration. As an outcome, miR-16-5p was selected out after Venn diagram analysis in these two groups. Then, we further explored miR-16-5p expression in T1DM patients on the basis of data acquired from the GEO database (no. GSE97123) and a significantly low expression of miR-16-5p was found in T1DM tissues $(n=12)$ compared to control samples $(n=12)$ (Fig. 1C).

\section{MiR-16-5p is down-regulated in HG-treated $\beta$-TC-tet cells}

To study the impact of miR-16-5p in diabetes, we established a high glucose-induced $\beta$-TC-tet cell model in vitro. We discovered that the $\mathrm{OD}_{450}$ value was reduced under $16.7 \mathrm{mM}$ conditions at $48 \mathrm{~h}$ and $72 \mathrm{~h}$ when compared with $2.8 \mathrm{mM}$ condition and control groups (Fig. 2A), while the $\mathrm{OD}_{450}$ values in the $2.8 \mathrm{mM}$ and control groups showed no obvious difference (Fig. 2A). In order to further probe whether the changes in the proliferation of HG-treated $\beta$-TC-tet cell were related to miR-16-5p expression, we performed the following experiments. Figure $2 \mathrm{~B}$ suggested that miR-16-5p expression level in $16.7 \mathrm{mM}$ group was reduced compared with the control and $2.8 \mathrm{mM}$ groups, while the levels of miR-16-5p in the control and $2.8 \mathrm{mM}$ groups had no difference (Fig. 2B). These results implied that the model with $16.7 \mathrm{mM}$ was successful, while $2.8 \mathrm{mM}$ had little effect. Hence, we used $2.8 \mathrm{mM}$ as a control group in the following experiments. With the goal of investigating whether miR-16-5p was involved in HG-induced pancreatic cell injury, we tested the influence of miR-16-5p mimic or inhibitor on $\beta$-TC-tet cell proliferation, which was analysed by CCK8 assay. As illustrated in Figure $2 \mathrm{C}$, transfection of $\beta$-TC-tet cells with 


\section{A}

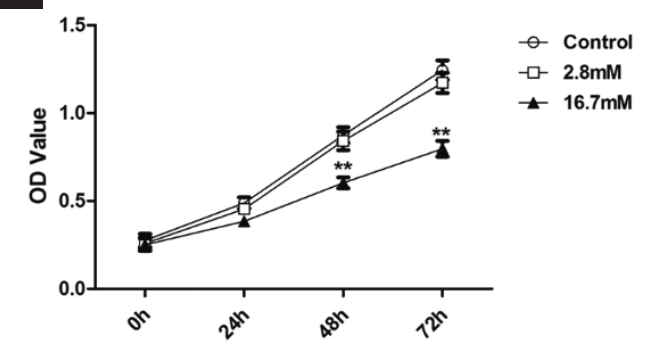

B

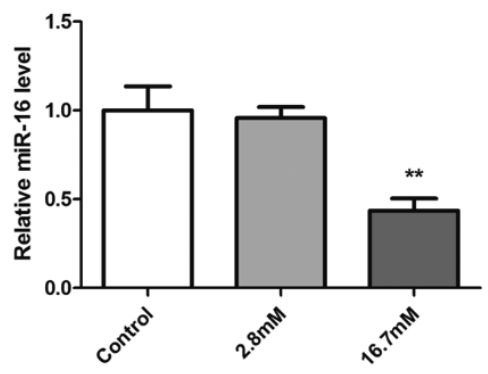

c

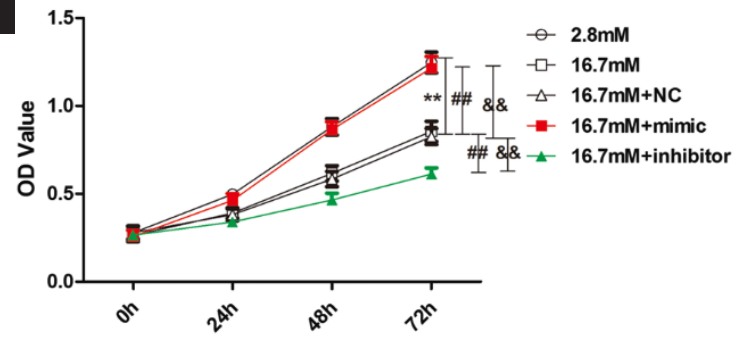

Figure 2. MiR-16-5p is down-regulated in HG-treated $\beta$-TC-tet cells. A. High glucose $(16.7 \mathrm{mM})$ decreased the proliferation of $\beta$-TC-tet cells, which was detected by CCK8. ${ }^{* *} p<0.01$ vs. control group. B. qRT-PCR analysis exhibited that miR-16-5p expression in $\beta$-TC-tet cells exposed with $16.7 \mathrm{mM}$ glucose was obviously decreased relative to control and $2.8 \mathrm{mM}$ glucose. ${ }^{* *} p<0.01 \mathrm{vs}$. control group. C. CCK8 assay showed that the transfection of miR16-5p mimic or inhibitor significantly accelerated or reduced the proliferation of $\beta$-TC-tet cells. ${ }^{* *} p<0.01$ vs. $2.8 \mathrm{mM}$ group, ${ }^{\# \# ~} p$ $<0.01$ vs. $16.7 \mathrm{mM}$ group, ${ }^{\&} \mathrm{~s} p<0.01$ vs. $16.7 \mathrm{mM}+$ NC group

miR-16-5p mimic distinctly reversed the suppression of cell proliferation caused by treatment with $16.7 \mathrm{mM}$ glucose, whereas miR-16-5p inhibitor further restrained the proliferation of $\beta$-TC-tet cells compared with the $16.7 \mathrm{mM}$ and $16.7 \mathrm{mM}+\mathrm{NC}$ groups, suggesting that $\beta$-TC-tet cells transfected with miR-16-5p mimic had the capacity to defend against HG-induced injury. These findings suggest that HG might stimulate cytotoxicity via down-regulating the expression of miR-16-5p in $\beta$-TC-tet cells.

\section{MiR-16-5p is an upstream miRNA of CXCL10, and CXCL10 is negatively modulated by $\operatorname{miR}-16-5 p$}

To further confirm the interaction between miR-16-5p and CXCL10 revealed by bioinformatics analysis, we further performed a luciferase reporter assay. The potential binding sites between miR-16-5p and CXCL10 predicted by Targetscan software are presented in Figure 3A. Cells co-transfected with miR-16-5p mimic and CXCL10-WT displayed an obvious decrease in luciferase activity, affirming the target relationship between CXCL10 and miR-16-5p, while miR-16-5p inhibitor increased the luciferase activity of CXCL10-WT (Fig. 3B). In addition, the co-transfection of CXCL10-MUT and miR-16-5p mimic/inhibitor had little impact on the luciferase activities (Fig. 3B). To confirm the negative relationship between CXCL10 and miR-16-5p, qRT-PCR and western blotting were carried out. The mRNA and protein levels of CXCL10 were remarkably down-regulated by miR-16-5p high expression, while miR-16-5p depletion acted the opposite effects on either mRNA or protein level of CXCL10. Above all, miR-16-5p was predicted as an upstream miRNA of CXCL10, and CXCL10 was negatively modulated by miR-16-5p.

\section{CXCL10 and miR-16-5p are associated with the proliferation and apoptosis}

\section{of HG-induced $\beta$-TC-tet cells}

To explore the influences of CXCL10 and miR-16-5p on the proliferation and apoptosis of $\beta$-TC-tet cells in the present of HG stimulation, CCK8 and flow cytometry assays were executed. As shown in Figure 4A, the fortifying impact of miR-16-5p over-expression on HG-induced $\beta$-TC-tet cell proliferation was obviously decreased by CXCL10 over-expression. Also, the suppressive influence of miR-16-5p ablation on the proliferation of HG-treated $\beta$-TC-tet cells was significantly reversed by CXCL10 silencing (Fig. 4B). Moreover, the restraining influence of miR-16-5p up-regulation on the apoptosis of HG-stimulated $\beta$-TC-tet cells was inversed by CXCL10 over-expression (Fig. 4C-D), while the facilitating influence of miR-16-5p deficiency on the apoptosis of HG-exposed $\beta$-TC-tet cells was distinctly reduced by CXCL10 knockdown (Fig. 4E-F). Then we used western blotting to ulteriorly determine the impacts of CXCL10 and miR-16-5p on the apoptotic signals of HG-induced $\beta$-TC-tet cells. As illustrated in Figure $4 \mathrm{G}-\mathrm{H}$, after HG-treated $\beta$-TC-tet cells were transfected with miR-16-5p mimic, the original lessened expression of Bcl-2 was increased whereas the initial elevated expression levels of Bax and Cleaved Caspase-3 were downregulated. CXCL10 over-expression clearly reduced the expression of Bcl-2 but elevated Bax and Cleaved Caspase-3 levels (Fig. 4G-H). Furthermore, CXCL10 silencing effectively reversed the change trend of Bcl-2, Bax, and Cleaved Caspase-3 expression caused by miR-16-5p; however, there was no significant difference in Caspase-3 expression (Fig. 4I-J). Therefore, CXCL10 interference reversed the influences of 


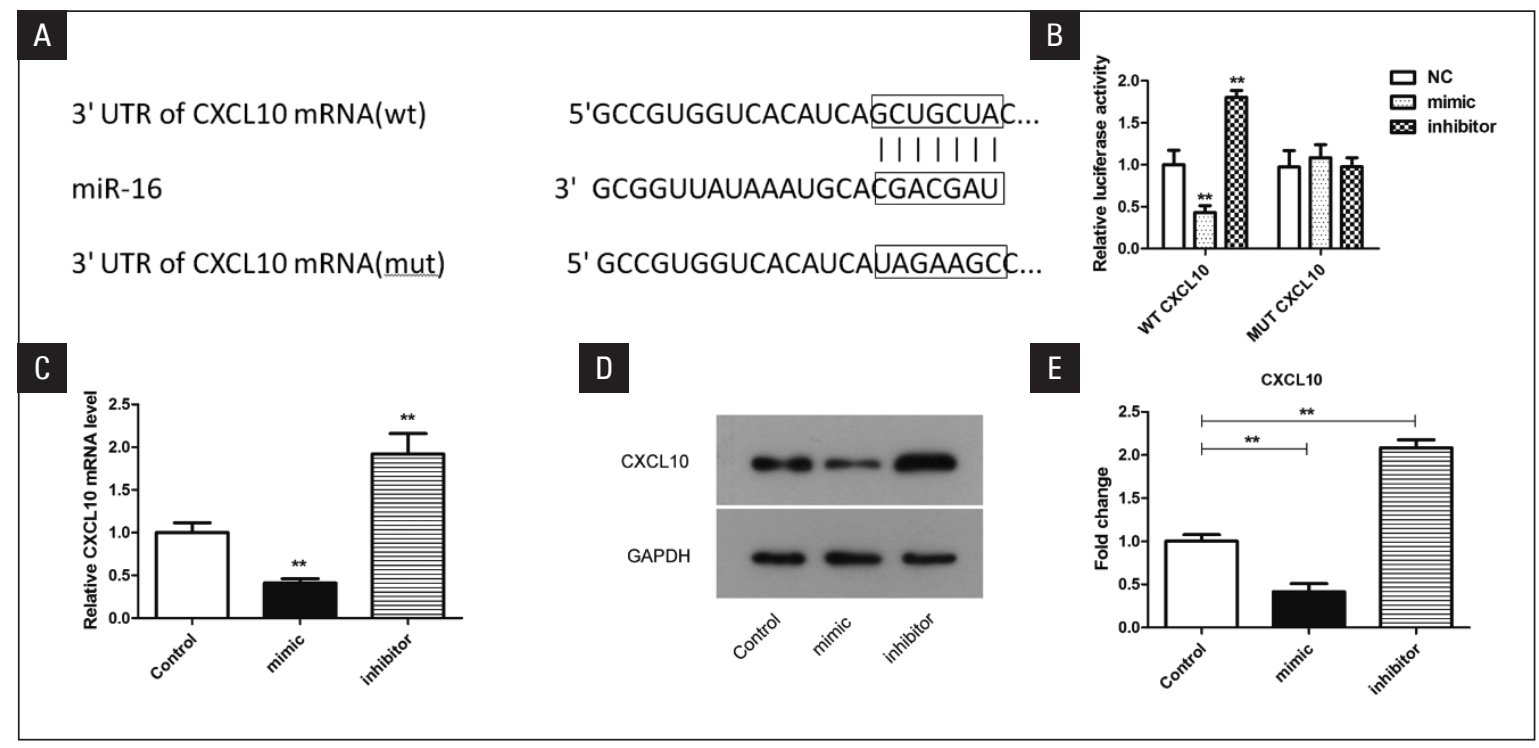

Figure 3. MiR-16-5p is an upstream miRNA of CXCL10, and CXCL10 is negatively modulated by miR-16-5p. A. A binding site between miR-16-5p and 3'-UTR of CXCL10 was predicted by the Targetscan website. B. MiR-16-5p mimic obviously reduced while miR-16-5p inhibitor fortified the luciferase activity of pGL3-CXCL10-3'-UTR (WT), but miR-16-5p mimic or inhibitor did not affect the luciferase activity of the pGL3-CXCL10-3'-UTR (MUT) group. C. qRT-PCR analysis indicated that CXCL10 is negatively modulated by miR16-5p. D, E. The results of western blotting demonstrated that CXCL10 protein level was dramatically decreased after transfection of miR-16-5p mimic and fortified by miR-16-5p inhibitor. ${ }^{* *} p<0.01$ vs. NC or control group

miR-16-5p interference on the proliferation and apoptosis of HG-induced $\beta$-TC-tet cells.

\section{Discussion}

As an insulin-dependent type of diabetes, T1DM is relatively harmful to human health [20]. Autoimmune-mediated pancreatic $\beta$-cell destruction is one of the pivotal pathophysiological mechanisms of T1DM, leading to severe insulin deficiency [21, 22]. CXCL10 has been reported to be highly expressed in the tissues of T1DM [15]. However, there are few related studies on the upstream regulatory factors (which also play key roles in T1DM) of this gene. In our study, we predicted and proved that miR-16-5p was an upstream miRNA that negatively modulated CXCL10 in pancreatic $\beta$-cells. Also, we found that CXCL10 was highly and miR-16-5p was lowly expressed in T1DM patients. More importantly, miR-16-5p modulated the proliferation and apoptosis of HG-induced $\beta$-TC-tet cells through targeting of CXCL10.

Studies have shown that CXCL10 and its receptor CXCR3 are concerned with the occurrence and development of diabetes (especially for T1DM) [23-25]. In animal models of diabetes, $\beta$ cells synthesise and secrete CXCL10, which is the original motive force attracting CXCR3 T cells to infiltrate the islet $[26,27]$. Therefore, the specific expression of CXCL10 in diabetic individuals can be regarded as one of the important factors for the pathogenesis of diabetes. However, there are few related studies on the upstream regulatory factors of this gene playing key roles in T1DM.

By utilising bioinformatics analysis and luciferase reporter assay, miR-16-5p was identified and confirmed as a candidate miRNA that might bind to CXCL10. The mechanisms by which miRNAs apply their influences are multitudinous; among them, miRNAs wielding as an upstream regulatory miRNA to modulate genes is momentous $[28,29]$. Prior work has indicated that miR-16-5p over-expression reduced the level of ANXA11 in hepatocellular carcinoma cells [30]. Furthermore, it is noteworthy that miR-16-5p was dysregulated in T1DM patients through bioinformatics analysis [19]. Until now, little information on the effects of miR-16-5p in T1DM has reported, which leads to increased interest in studying the impact of miR-16-5p on the development of T1DM.

Our data point to a regulatory impact of miR-16-5p on the proliferation and apoptosis of HG-induced $\beta$-TC-tet cells, which was partly reflected by CXCL10. Under the impact of T-helper (Th) 1 cytokines, $\beta$-cells could produce CXCL10, and then further suppress its proliferation in T1DM [23]. It was revealed that CXCL10 induced the proliferation of pancreatic $\beta$ cells and mediated the apoptosis of pancreatic $\beta$ cells [31].

As reported, miR-16-5p facilitated the proliferation and metastasis, and suppressed the apoptosis in hepatocellular carcinoma cells through targeting of ANXA11 [30]. Directly, miR-16-5p was demonstrated to target SESN1 to affect the proliferation and apoptosis 
A

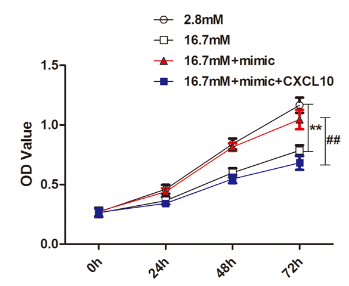

C

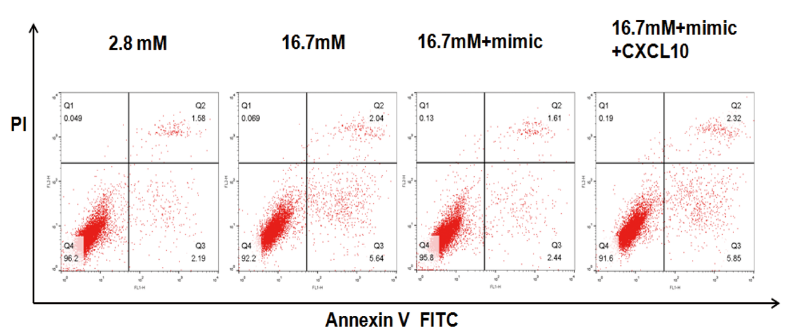

E

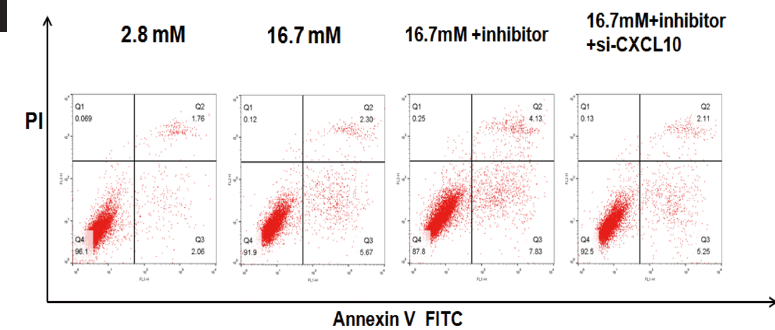

B

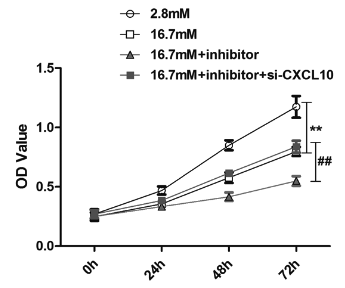

D

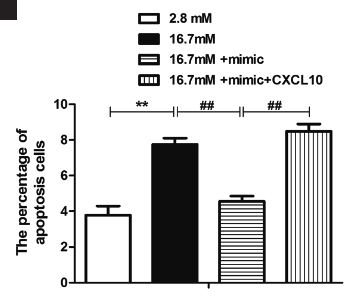

$\mathrm{F}$

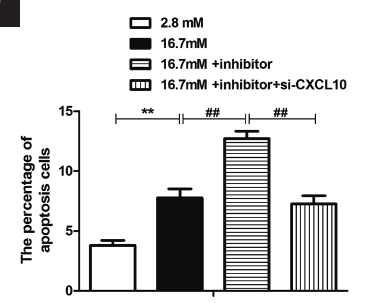

H

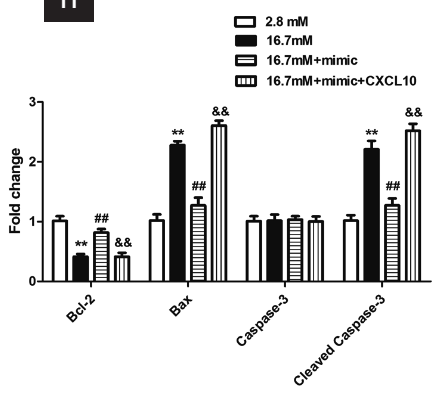

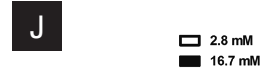

互 $\begin{aligned} & 16.7 \mathrm{mM} \\ & 16.7 \mathrm{mM} \text { tinnhibitor }\end{aligned}$

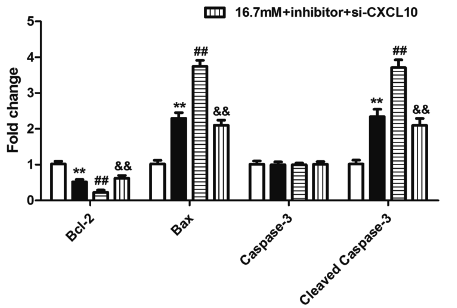

G

I
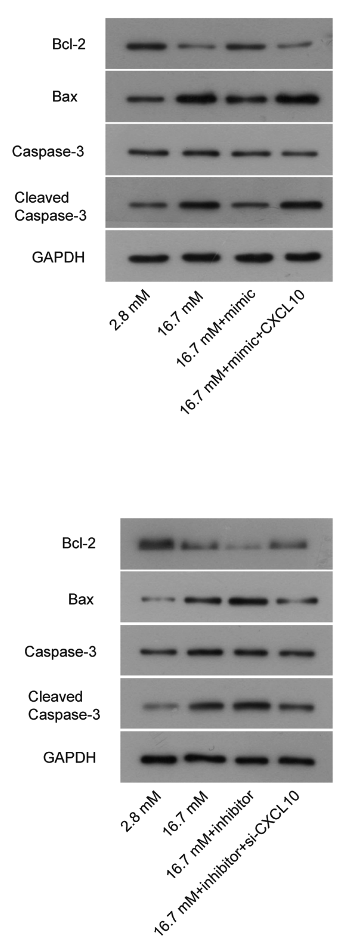

Figure 4. CXCL10 reversed the influences of miR-16-5p on the proliferation and anti-apoptosis of HG-induced $\beta$-TC-tet cells. A. The proliferation of HG-induced $\beta$-TC-tet cells following treatment with miR-16-5p mimic, miR-16-5p mimic + pcDNA3.1-CXCL10, which were tested by CCK8. B. Cell proliferation ability of HG-treated $\beta$-TC-tet cells following treatment with miR-16-5p inhibitor, miR-16-5p inhibitor + si-CXCL10, which were measured using CCK8 assay. C-F. Cell apoptosis was tested by flow cytometry analysis. (G-J) The protein levels of Bcl-2, Bax, Caspase-3, and Cleaved Caspase-3. ${ }^{* *} p<0.01$ vs. $2.8 \mathrm{mM}$ group, ${ }^{\# \#} p<0.01$ vs. $16.7 \mathrm{mM}$ group, ${ }^{\& \&} p<0.01$ vs. $16.7 \mathrm{mM}+$ mimic or $16.7 \mathrm{mM}+$ inhibitor group 
of myoblast [32]. In traumatic brain injury patients, a mechanism for $\mathrm{Bcl}-2$ regulation via miR-16-5p in the proliferation and apoptosis of osteoblasts was observed [33]. Moreover, high expression of miR-16-5p inhibitor obviously weakened the apoptosis and cleaved caspase-3 stimulated by PVT1 silencing in renal carcinoma cells [34]. In line with the above reports, we found that CXCL10 could partly reverse the regulatory impact of miR-16-5p on the proliferation and anti-apoptosis of HG-treated $\beta$-TC-tet cells.

\section{Conclusions}

To conclude, our results provide promising evidence that miR-16-5p may modulate $\beta$-TC-tet cell proliferation and apoptosis through targeting of CXCL10. Of note, miR-16-5 $p$ was predicted as an upstream regulatory miRNA that regulates CXCL10 in $\beta$-TC-tet cells, for the first time. Extensive study of the potential impact of the mechanism of miR-16-5p on T1DM will lay a solid foundation for T1DM diagnosis and treatment.

\section{References}

1. Yi Bo, Huang G, Zhou Z. Different role of zinc transporter 8 between type 1 diabetes mellitus and type 2 diabetes mellitus. J Diabetes Investig. 2016; 7(4): 459-465, doi: 10.1111/jdi.12441, indexed in Pubmed: 27181765.

2. Novotna M, Podzimek S, Broukal Z, et al. Periodontal Diseases and Dental Caries in Children with Type 1 Diabetes Mellitus. Mediators Inflamm. 2015; 2015: 379626, doi: 10.1155/2015/379626, indexed in Pubmed: 26347009

3. Rak K, Bronkowska M. Immunomodulatory Effect of Vitamin D and Its Potential Role in the Prevention and Treatment of Type 1 Diabetes Mellitus-A Narrative Review. Molecules. 2018; 24(1), doi: 10.3390/molecules24010053, indexed in Pubmed: 30586887.

4. Zhang L, Zhang Q. Glycated Plasma Proteins as More Sensitive Markers for Glycemic Control in Type 1 Diabetes. Proteomics Clin Appl. 2020; 14(2): e1900104, doi: 10.1002/prca.201900104, indexed in Pubmed: 31868294.

5. Chellappan DK, Sivam NS, Teoh KX, et al. Gene therapy and type 1 diabetes mellitus. Biomed Pharmacother. 2018; 108: 1188-1200, doi: 10.1016/j.biopha.2018.09.138, indexed in Pubmed: 30372820.

6. Fu S, Li L, Deng S, et al. Effectiveness of advanced carbohydrate counting in type 1 diabetes mellitus: a systematic review and meta-analysis. Sci Rep. 2016; 6: 37067, doi: 10.1038/srep37067, indexed in Pubmed: 27841330.

7. Bjerg L, Hulman A, Charles M, et al. Clustering of microvascular complications in Type 1 diabetes mellitus. J Diabetes Complications. 2018; 32(4): 393-399, doi: 10.1016/j.jdiacomp.2018.01.011, indexed in Pubmed: 29478814

8. Garcia-Contreras M, Brooks RW Boccuzzi L et al. Exosomes as biomarkers and therapeutic tools for type 1 diabetes mellitus. Eur Rev Med Pharmacol Sci. 2017; 21(12): 2940-2956, indexed in Pubmed: 28682421.

9. He J, Lian C, Fang Y, et al. Effect of CXCL10 receptor antagonist on islet cell apoptosis in a type I diabetes rat model. Int J Clin Exp Pathol. 2015; 8: 14542-14548, indexed in Pubmed: 26823775.

10. Lee JH, Kim B, Jin WJ, et al. Pathogenic roles of CXCL10 signaling through CXCR3 and TLR4 in macrophages and T cells: relevance for arthritis. Arthritis Res Ther. 2017; 19(1): 163, doi: 10.1186/s13075-017-1353-6, indexed in Pubmed: 28724396.

11. Fan $Y$, Zhang W, Wei H, et al. Hepatic NK cells attenuate fibrosis progression of non-alcoholic steatohepatitis in dependent of CXCL10-mediated recruitment. Liver Int. 2020; 40(3): 598-608, doi: 10.1111/liv.14307, indexed in Pubmed: 31758647.

12. Antonelli A, Ferrari SM, Giuggioli D, et al. Chemokine (C-X-C motif) ligand (CXCL)10 in autoimmune diseases. Autoimmun Rev. 2014; 13(3): 272-280, doi: 10.1016/j.autrev.2013.10.010, indexed in Pubmed: 24189283.

13. Chen Ju, Ye X, Pitmon E, et al. IL-17 inhibits CXCL9/10-mediated recruitment of CD8 cytotoxic $\mathrm{T}$ cells and regulatory $\mathrm{T}$ cells to colorectal tumors. J Immunother Cancer. 2019; 7(1): 324, doi: 10.1186/s40425-019-0757-z, indexed in Pubmed: 31775909.
14. Homann D. Back From the Brink: The Uses of Targeting the CXCL10:CXCR3 Axis in Type 1 Diabetes. Diabetes. 2015; 64(12): 3990-3992, doi: 10.2337/dbi15-0019, indexed in Pubmed: 26604174.

15. Ahmadi Z, Arababadi MK, Hassanshahi G. CXCL10 activities, biological structure, and source along with its significant role played in pathophysiology of type I diabetes mellitus. Inflammation. 2013; 36(2): 364-371, doi: 10.1007/s10753-012-9555-1, indexed in Pubmed: 23065240.

16. Pan W, Zhang Y, Zeng C, et al. miR-192 is upregulated in T1DM, regulates pancreatic -cell development and inhibits insulin secretion through suppressing GLP-1 expression. Exp Ther Med. 2018; 16(3): 2717-2724 doi: 10.3892/etm.2018.6453, indexed in Pubmed: 30186503.

17. Lakhter AJ, Pratt RE, Moore RE, et al. Beta cell extracellular vesicle miR-21-5p cargo is increased in response to inflammatory cytokines and serves as a biomarker of type 1 diabetes. Diabetologia. 2018; 61(5): 1124-1134, doi: 10.1007/s00125-018-4559-5, indexed in Pubmed: 29445851.

18. Assmann TS, Recamonde-Mendoza M, De Souza BM, et al. MicroRNA expression profiles and type 1 diabetes mellitus: systematic review and bioinformatic analysis. Endocr Connect. 2017; 6(8): 773-790, doi: 10.1530/EC-17-0248, indexed in Pubmed: 28986402.

19. Assmann TS, Recamonde-Mendoza M, Costa AR, et al. Circulating miRNAs in diabetic kidney disease: case-control study and in silico analyses. Acta Diabetol. 2019; 56(1): 55-65, doi: 10.1007/s00592-018-1216-x, indexed in Pubmed: 30167868

20. Yue Y, Tang Y, Tang J, et al. Maternal infection during pregnancy and type 1 diabetes mellitus in offspring: a systematic review and meta-analysis. Epidemiol Infect. 2018; 146(16): 2131-2138, doi: 10.1017/S0950268818002455, indexed in Pubmed: 30152300.

21. Herold KC, Vignali DAA, Cooke A, et al. Type 1 diabetes: translating mechanistic observations into effective clinical outcomes. Nat Rev Immunol. 2013; 13(4): 243-256, doi: 10.1038/nri3422, indexed in Pubmed: 23524461.

22. DiMeglio LA, Evans-Molina C, Oram RA. Type 1 diabetes. Lancet. 2018 391(10138): 2449-2462, doi: 10.1016/S0140-6736(18)31320-5, indexed in Pubmed: 29916386

23. Antonelli A, Ferrari SM, Corrado A, et al. CXCR3, CXCL10 and type 1 diabetes. Cytokine Growth Factor Rev. 2014; 25(1): 57-65, doi: 10.1016/j. cytogfr.2014.01.006, indexed in Pubmed: 24529741.

24. Homann D. Back From the Brink: The Uses of Targeting the CXCL10:CXCR3 Axis in Type 1 Diabetes. Diabetes. 2015; 64(12): 3990-3992, doi: 10.2337/dbi15-0019, indexed in Pubmed: 26604174

25. Corrado A, Ferrari SM, Ferri C, et al. Type 1 diabetes and (C-X-C motif) ligand (CXCL) 10 chemokine. Clin Ter. 2014; 165(2): e181-e185, doi: 10.7471/CT.2014.1706, indexed in Pubmed: 24770831.

26. Christen S, Holdener M, Beerli C, et al. Small molecule CXCR3 antagonist NIBR2130 has only a limited impact on type 1 diabetes in a virus-induced mouse model. Clin Exp Immunol. 2011; 165(3): 318-328, doi: 10.1111/j.1 365-2249.2011.04426.x, indexed in Pubmed: 21649647.

27. Coppieters KT, Amirian N, Pagni PP, et al. Functional redundancy of CXCR3/CXCL10 signaling in the recruitment of diabetogenic cytotoxic T lymphocytes to pancreatic islets in a virally induced autoimmune diabetes model. Diabetes. 2013; 62(7): 2492-2499, doi: 10.2337/db12-1370, indexed in Pubmed: 23434930.

28. Liu B, Shyr Yu, Cai J, et al. Interplay between miRNAs and host genes and their role in cancer. Brief Funct Genomics. 2018; 18(4): 255-266, doi: 10.1093/bfgp/elz002, indexed in Pubmed: 30785618.

29. Steiman-Shimony A, Shtrikman O, Margalit H. Assessing the functional association of intronic miRNAs with their host genes. RNA. 2018; 24(8): 991-1004, doi: 10.1261/rna.064386.117, indexed in Pubmed: 29752351.

30. Liu Z, Wang Y, Wang L, et al. Long non-coding RNA AGAP2-AS1, functioning as a competitive endogenous RNA, upregulates ANXA11 expression by sponging miR-16-5p and promotes proliferation and metastasis in hepatocellular carcinoma. J Exp Clin Cancer Res. 2019; 38(1): 194, doi: 10.1186/s13046-019-1188-x, indexed in Pubmed: 31088485.

31. Schulthess FT, Paroni F, Sauter NS, et al. CXCL10 impairs beta cell function and viability in diabetes through TLR4 signaling. Cell Metab. 2009; 9(2): 125-139, doi: 10.1016/j.cmet.2009.01.003, indexed in Pubmed: 19187771

32. Cai B, Ma M, Chen B, et al. MiR-16-5p targets SESN1 to regulate the p53 signaling pathway, affecting myoblast proliferation and apoptosis, and is involved in myoblast differentiation. Cell Death Dis. 2018; 9(3): 367, doi: 10.1038/s41419-018-0403-6, indexed in Pubmed: 29511169.

33. Sun Y, Xiong Y, Yan C, et al. Downregulation of microRNA-16-5p accelerates fracture healing by promoting proliferation and inhibiting apoptosis of osteoblasts in patients with traumatic brain injury. Am J Transl Res. 2019; 11(8): 4746-4760, indexed in Pubmed: 31497196.

34. Ren Yu, Huang W, Weng G, et al. LncRNA PVT1 promotes proliferation, invasion and epithelial-mesenchymal transition of renal cell carcinoma cells through downregulation of miR-16-5p. Onco Targets Ther. 2019; 12: 2563-2575, doi: 10.2147/OTT.S190239, indexed in Pubmed: 31040699. 